\title{
Oil-in-water fL droplets by interfacial spontaneous fragmentation and their electrical characterization
}

\author{
Giuseppe Arrabito, ${ }^{*}$ a Vito Errico, ${ }^{b, c}$ Adele De Ninno,,${ }^{b, d}$ Felicia Cavaleri, ${ }^{a}$ Vittorio Ferrara, ${ }^{e}$ \\ Bruno Pignataro, ${ }^{a}$ and Federica Caselli ${ }^{*}, b$ \\ ${ }^{a}$ Department of Physics and Chemistry, University of Palermo, Palermo, Italy \\ ${ }^{\mathrm{b}}$ Department of Civil Engineering and Computer Science, University of Rome Tor Vergata, \\ Roma, Italy \\ ${ }^{c}$ Department of Electronic Engineering, University of Rome Tor Vergata, Roma, Italy \\ ${ }^{\mathrm{d}}$ Institute for Photonics and Nanotechnologies, Italian National Research Council, Roma, Italy. \\ ${ }^{\mathrm{e}}$ Department of Chemical Sciences, University of Catania, Catania, Italy.
}

KEYWORDS. Inkjet printing, Oil-in-water emulsion, Electrical characterization, Single-particle analysis.

ABSTRACT. Inkjet printing is here employed for the first time as a method to produce femtoliter (fL) scale oil droplets dispersed in water. In particular, picoliter (pL) scale fluorinated oil (FC40) droplets are printed in presence of perfluoro-1-octanol (PFCO) surfactant at velocity higher than $5 \mathrm{~m} / \mathrm{s}$. Femtoliter scale oil droplets in water are spontaneously formed through a fragmentation process at the water/air interface by using minute amounts of non-ionic surfactant (down to $0.003 \% \mathrm{v} / \mathrm{v}$ of Tween 80 ). This fragmentation occurs by a Plateau-Rayleigh mechanism at moderately high Weber number $\left(10^{1}\right)$. A microfluidic chip with integrated microelectrodes allows droplet characterization in terms of number and diameter distribution (peaked at about 3 microns) by means of electrical impedance measurements. These results show an unprecedented possibility to scale-up oil droplets down to the femtoliter scale which opens up several perspectives for a tailored oil-in-water emulsions fabrication for drug encapsulation, pharmaceutic preparations and cellular biology.

\section{Introduction}

Emulsions can be defined as a fine dispersion of minute micro-droplets of one liquid (the dispersed phase) in another (the continuous phase) in which the dispersed phase is not soluble or miscible. ${ }^{1}$ They are kinetically stabilized by the presence of surfactants to reduce the interfacial tension between the two immiscible layers. ${ }^{2}$ Emulsified droplets are the basis for many systems employed in food science, ${ }^{3}$ biological chemistry, ${ }^{4}$ pharmaceutical chemistry, ${ }^{5}$ drug delivery 6,7 
platforms for artificial life ${ }^{8-10}$. In particular, droplets at the micron size have emerged as an important system for these applications, given the easiness to finely adapt their physicochemical bulk/surface composition ${ }^{7,11}$ and their properties to the system of interest. The mild generation of oil droplets at micron size along with the characterization of their properties as single and as collective systems is consequently a topic of great interest.

Oil droplets emulsions are conventionally prepared by employing colloid mills and highpressure homogenizers, ${ }^{5}$ based on the application of high shear to break-up large emulsion droplets into smaller ones that are stabilized by emulsifier. This results in poor control on droplet size, with coefficient of variation $(\mathrm{CV}$ - i.e. the percentage measure of dispersion of the droplet size around the average value, calculated as the ratio of the standard deviation to the mean droplet size) of typically $40 \% .{ }^{12}$ Research efforts have focused on the fabrication of emulsification methods at low energy consumption such as membrane and microfluidics emulsification. ${ }^{13}$ In membrane emulsification, the dispersed phase is extruded through micro pores into a flowing continuous phase. Oil droplets with a CV of diameter reaching $10 \%$ can be produced. ${ }^{14}$ The uniformity of the droplets sizes depends on the viscosity of the dispersed phase and on the size distribution of the membrane pores. ${ }^{15}$ On the other hand, microfluidic emulsification devices can be divided in shear-based ${ }^{16,17}$ and spontaneous or interfacial tension driven approaches. ${ }^{18}$ In shear-based systems, the flow of the dispersed and continuous phases influences the droplet size. In spontaneous microfluidics emulsification devices, the drop formation is driven only by interfacial tension forces. ${ }^{19,20}$ However, the flow of the continuous phase is necessary to avoid droplets crowding at the nozzles, ${ }^{14}$ finally creating polydispersity and introducing mechanical stresses. An alternative to microfluidics setups is constituted by interfacial emulsification, in which droplets formation at the air-liquid interface of the continuous phase is based on interfacial shearing. ${ }^{21}$ However, the smallest droplet volume is limited to tens of picoliters.

In this scenario, alternative and customizable approaches to fabricate oil droplets emulsified in water are needed. Possible solutions can be provided by printing approaches. ${ }^{22-25}$ Among them, inkjet printing is a convenient choice in terms of compatibility to liquids with different viscosities and densities (aqueous, organic, polymeric, oil). ${ }^{22,26}$ For instance, picoliter scale droplets by inkjet printing can be employed for detecting few molecules ${ }^{27,28}$ and studying enzymatic assays. ${ }^{29}$ As to oil in water emulsions, Zeng et.al ${ }^{30}$ showed the possibility to produce oil droplets in water $(\mathrm{O} / \mathrm{W})$ in a range of hundreds of microns $(210-290 \mu \mathrm{m})$. In their experiments, the nozzles print-head was immersed in water, this potentially leading to printhead contamination due to the contact between nozzles and the aqueous phase. In order to solve this problem, oil droplets should be immersed into the aqueous phase without printhead/water phase contact. Accordingly, the impact of a macroscopic drop onto a bath of an immiscible liquid results in a complex dynamics, which can lead to the tailored production of oil-in-water capsules with ultra-thin polymeric sheets, ${ }^{11}$ the spontaneous formation of Marangoni flows in case of a aqueous phase enriched with surfactants ${ }^{31}$ or drop fragmentation into a collection of non-coalescing smaller daughter drops. ${ }^{32}$ The dynamics of oil-in-water droplet encapsulation depends upon the impacting droplet Weber number $\left(\mathrm{We}=\rho \mathrm{V}^{2} \mathrm{~d}_{0} / \sigma\right.$ where $\rho\left[\mathrm{Kg} / \mathrm{m}^{3}\right]$ is the density, $\mathrm{V}\left[\mathrm{m} / \mathrm{s}^{2}\right]$ is the velocity, $\mathrm{d}_{0}[\mathrm{~m}]$ is the diameter and $\sigma[\mathrm{N} / \mathrm{m}]$ is the surface energy). If a macroscopic droplet has a sufficient kinetic energy ${ }^{33}$ (We in order of $10^{1}$ ), after an initial spreading on the water surface, it can be immersed in the aqueous phase. In addition, spontaneous Plateau-Rayleigh instabilities in the oil droplet spreading at the water surface can 
lead to the droplet fragmentation into smaller daughter droplets which can be immersed in the aqueous phase. However, the dynamics of picoliter-scale oil droplet impact when at interface with aqueous phase is unknown.

In order to understand and optimize droplet production processes, effective methods for detection and counting of oil droplets dispersed in the aqueous phase down to the single droplet level are required. Optical investigation can be used, which however is time consuming and operator-dependent. As an alternative, a faster and automated approach is constituted by microfluidic impedance cytometry, which is a label-free technique for high-throughput electrical characterization of single particles and cells. ${ }^{34-37}$ A microfluidic impedance chip typically consists of a microchannel equipped with microelectrodes and filled with a conductive buffer. A stimulation voltage is applied to a pair of electrodes and the differential current collected from another electrode pair is measured. The signal trace recorded upon the passage of a flowing particle conveys information on particle geometric and dielectric properties. In particular, for insulating particles, signal amplitude is proportional to particle volume. Moreover, this approach is recently gaining attention as a characterization and monitoring tool for digital and droplet microfluidics. ${ }^{38-40}$

In this work, we show for the first time that picoliter scale oil droplets produced by inkjet printing can be successfully encapsulated in aqueous phases and, in presence of PlateauRayleigh instabilities at the oil/water interface, they can produce daughter droplets at the femtoliter scale. Given the significant difference in resistivity between the oil and the aqueous phase, automated counting and sizing at the single droplet level is performed within a microfluidic impedance chip. Our results can be considered as an unprecedented effective and customizable approach to micron-sized oil droplets production and characterization.

\section{Materials and Methods}

Chemicals. Oil-in-water emulsions were fabricated on 8-well Chambered Coverglass with nonremovable wells (Corning, No. 1 borosilicate glass bottom). FC40 fluorinated oil (3M $\mathrm{M}^{\mathrm{TM}}$ Fluorinert $^{\mathrm{TM}}$ Electronic Liquid FC-40), 1H,1H,2H,2H-Perfluoro-1-octanol 97\% (Sigma Aldrich), $\mathrm{NaCl}$, Tris (2-Amino-2-(hydroxymethyl)-1,3-propanediol), EDTA ((Ethylenedinitrilo)tetraacetic acid), Tween 80 (Polyethylene glycol sorbitan monooleate), Sucrose (for molecular biology, $\geq 99.5 \%$ ), Atto655 biotin (BioReagent, suitable for fluorescence, Sigma Aldrich), Polystyrene beads (Polysciences) were purchased and used as received.

Oil-in-water droplets fabrication. The experimental setup used for the fabrication of the oil droplets is shown in Figure S1. All aqueous solutions were prepared in ultrapure Millipore water (Direct Q-UV filtration system, $18.2 \mathrm{M} \Omega \mathrm{cm})$. The $\mathrm{pH}$ was controlled by a $\mathrm{pH}$ meter $(\mathrm{pH}$ 700, Eutech Instruments). Picoliter-scale oil droplets were dispensed by using a Dimatix Materials Printer (DMP-2800, Fuji Film, Figure S1A) at 40\% Relative Humidity. This instrument was equipped with user fillable piezo-driven inkjet print cartridges, each with 16 nozzles $254 \mu \mathrm{m}$ spaced and $21.5 \mu \mathrm{m}(10 \mathrm{pL})$ or $10.5 \mu \mathrm{m}(1 \mathrm{pL})$ in diameter. Droplets were typically emitted at a jetting frequency of $3-5 \mathrm{kHz}$ with typical flow rates of $10 \mathrm{pL} / 10 \mu$ s (i.e. 1 $\mu \mathrm{L} / \mathrm{s}$ ) (considering the time necessary for the formation of a single droplet). FC40 ink droplets (FC40 added with $1.5 \%$ of the surfactant $1 \mathrm{H}, 1 \mathrm{H}, 2 \mathrm{H}, 2 \mathrm{H}$-perfluoro-1-decanol) were printed in the form of a dots array (361x321 with a drop-to-drop distance equal to $25 \mu \mathrm{m})$ inside $300 \mu \mathrm{L}$ of 
aqueous solution (deposited on each microwell - see Figure S1B) containing NaCl $300 \mathrm{mM}$, Tris $10 \mathrm{mM}$, EDTA $1 \mathrm{mM}$, Sucrose 30\%, pH 7.5, at different concentrations of Tween 80 (in the range comprised between $3 \%$ and $0.00003 \%$ v/v). Four aliquots of oil-in-water dispersions (each of $300 \mathrm{uL}$ ) were collected to get a sample of $1.2 \mathrm{~mL}$ to be used for electrical characterization. Before the impedance measurements, the sample was spiked with $6 \mu \mathrm{m}$ polystyrene beads to be used for calibration of the impedance chip $(0.012 \mathrm{~mL}$ of bead suspension in $1.2 \mathrm{~mL}$ of oil-inwater dispersion; bead suspension made of PBS $+0.1 \%$ Tween $20+130 \mathrm{mg} / \mathrm{mL}$ sucrose $+10^{7}$ beads $/ \mathrm{mL})$.

Oil droplets impedance-based characterization. A schematic representation of the experimental setup used for the impedance-based characterization of the oil droplets is shown in Figure S2. A commercial microfluidic chip for electrical impedance spectroscopy of single particles/cells was used (EIS chip, Micronit). The chip is made of borosilicate glass and an inert interstitial layer of dry film resist. It has a straight through channel $28 \mu \mathrm{m}$ deep and $30 \mu \mathrm{m}$ wide. There are two sets of facing electrodes $(\mathrm{Pt})$. Electrode dimension along channel axis is $20 \mu \mathrm{m}$, with planar separation of $20 \mu \mathrm{m}$ and $28 \mu \mathrm{m}$ depth separation as based on channel depth. A commercial chip holder was used (Fluidic Connect PRO, Micronit). A syringe pump (Elite 11, Harvard Apparatus) operating at $10 \mu \mathrm{L} / \mathrm{min}$ was used for fluidic control. Electrical measurements were performed using an impedance spectroscope (HF2IS, Zurich Instruments, 14 bit $\mathrm{A} / \mathrm{D}$ conversion at $210 \mathrm{M}$ sample per second), along with a transimpedance amplifier (HF2TA, Zurich Instruments) for signal conditioning. As pictured in Figure 1A, an AC voltage signal was applied to the top electrodes and the differential current flowing through the bottom electrodes was demodulated and recorded. With this wiring scheme, the passage of a particle that is insulating with respect to the suspending medium (like an oil droplet or a polystyrene bead in a conductive aqueous buffer) is recorded as a pair of opposite peaks, ${ }^{41}$ as explained in Figure S3. Typical settings were: $4 \mathrm{~V}$ at $1 \mathrm{MHz}$ applied potential, 115 ksample per second readout rate, 20 $\mathrm{kHz}$ filter bandwidth). Collected data streams were processed using a custom Matlab script. Segmentation was first carried out, with a previously reported algorithm, ${ }^{42}$ aimed at extracting events from the data stream. For each detected event, feature extraction was then performed by fitting a bipolar Gaussian template (Figure 1B) to the recorded trace. In particular, the particle electrical diameter $D$ was computed as ${ }^{43} D=G a^{1 / 3}$, where $a$ is the amplitude control parameter and $G$ is a gain factor accounting for the electronic circuitry. The gain factor $\mathrm{G}$ is calibrated by means of polystyrene beads, as typically done in impedance cytometry for biological and biotechnological applications. ${ }^{44-46}$ More specifically, in this work, polystyrene beads are employed because of their favourable properties (high impedance, size monodispersity) which make them ideal candidates and widely used model systems for impedance calibration in aqueous based solutions at high electrical conductivity. Particle velocity $V$ was computed from the transit time $\delta$. 


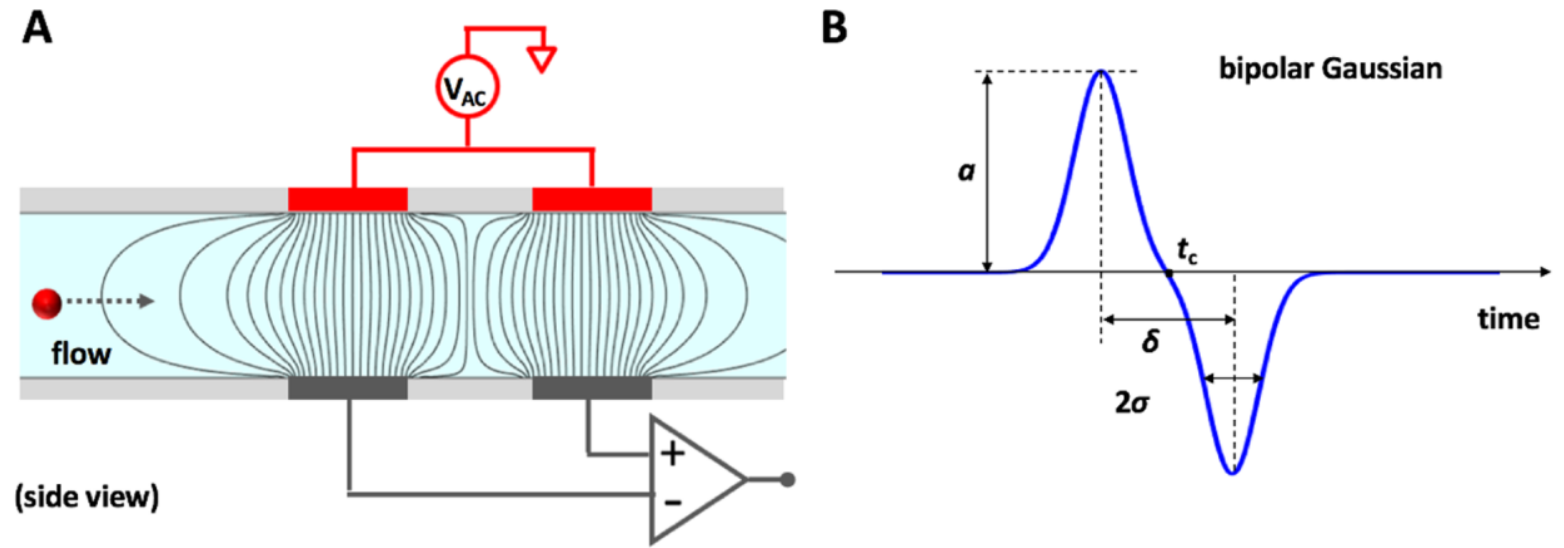

Figure 1. A) Schematic representation of the microfluidic impedance chip (side view) along with the relevant wiring scheme. An AC voltage is applied to the top electrodes and the differential current collected from the bottom electrodes is recorded. B) Bipolar Gaussian template used to fit the experimental traces.

Oil droplets optical visualization. The microfluidic chip allows for optical inspection. Images of the flowing droplets/beads were recorded using a high-speed camera (Photron FASTCAM Mini UX100, $4000 \mathrm{fps}, 4 \mu$ s shutter time) mounted on an inverted microscope (Zeiss Axio Observer, $20 \times$ objective).

\section{Results and Discussion}

\section{Oil-in-water droplets fabrication by inkjet printing}

It was initially developed a strategy to produce spherical and satellites-free FC40 picoliter scale oil droplets by optimizing the printing waveform (i.e. the voltage vs. time signal given as input to the piezoelectric actuator). In particular, mineral oil inks can have high viscosity at ambient conditions and their successful print is consequently possible only by employing a double pulse signal waveforms. ${ }^{28}$ On the other hand, the FC40 oil possesses a significantly lower viscosity (about $2 \mathrm{mPa} \cdot \mathrm{s}$ at $25^{\circ} \mathrm{C}$ ) which makes the resulting fluid to easily produce satellites if jetted under the conventional conditions (Figure S4), as for instance a double pulse signal waveforms. ${ }^{28}$ In order to solve this issue, a single pulse driving waveform was employed for the droplets ejection (Figure S5). In this signal pulse, the fall time $\left(t_{F}\right)$ is significantly increased, thus maximizing the negative pressure that pulls back the liquid thread towards the nozzle minimizing the occurrence of satellite droplets. The FC40 oil ink was spiked with $1.5 \% 1 \mathrm{H}, 1 \mathrm{H}, 2 \mathrm{H}, 2 \mathrm{H}-$ Perfluoro-1-octanol (PFCO) in order to facilitate the wetting of the nozzle surface. In fact, in the absence of surfactant, the FC40 oil droplets were not formed, but rather it was just pulsing at the nozzle exit. PFCO is a pseudosurfactant commonly employed to stabilize aqueous microdroplets in fluorous oils within poly(dimethylsiloxane) (PDMS) microdevices and does not affect surface tension value of FC40 oil. $^{47}$

To avoid satellite droplets, one has to keep the ejected liquid column exiting the nozzles as short as possible. Indeed, by using the proposed single pulse waveform at low jetting voltages (15-25 $\mathrm{V})$, it was possible to obtain almost spherical droplets with a very short tail which avoid formation of satellites (Figure 2A). Droplets produced by this waveform were characterized in 
the range of the jetting voltage comprised between $15 \mathrm{~V}$ and $35 \mathrm{~V}$ (Figure 2B). As expected, it was possible to observe that, at the same jetting voltage, droplet speed for $1 \mathrm{pL}$ droplets is slightly higher than the one of $10 \mathrm{pL}$, because of the lower size of the nozzle. ${ }^{29}$ As reported in the Supplementary Information (see Figure S6), FC40 oil droplets are characterized by a spherical shape and by the absence of satellites. In both cases, voltages higher than $25 \mathrm{~V}$ permit to obtain droplet speeds in the range $5-10 \mathrm{~m} / \mathrm{s}$ which is known to produce good droplet directionality ${ }^{48}$ to direct them into the aqueous phase filled microwell (see Figure S1).

Subsequently, pL-scale (1-10 pL) FC40 oil droplets spiked with perfluorooctanol surfactant $(1.5 \% \mathrm{v} / \mathrm{v})$ are printed inside the aqueous phase $(300 \mu \mathrm{L})$ manually deposited inside microwells (Figure S1). The resulting $300 \mu \mathrm{L}$ aliquots of oil-in-water emulsions are collected and are flown inside the microfluidic chip at controlled flow rate $(10 \mu \mathrm{L} / \mathrm{min})$. Note that the density of the FC40 oil is higher $\left(1855 \mathrm{~kg} / \mathrm{m}^{3}\right)$ with respect to the density of the aqueous phase (around 1130 $\mathrm{kg} / \mathrm{m}^{3}$ ), so that inkjetted oil droplets can be immersed into the aqueous phase. About 25 minutes are needed to print the array made of $361 \times 321$ droplets, which means that about 70-80 droplets are printed per second and that each new inkjetted droplet impacts the water surface after about 10 milliseconds from the previous one.

Once single pL scale FC40 droplets are immersed in the aqueous phase, they spread at the water/air interface and are subjected to capillary instabilities due to surface tension gradients between the droplets and the aqueous phase, which potentially leads to the production of smaller daughter droplets whose size is proportional to the amplitude of the largest capillary wave. ${ }^{33}$ It is known that simultaneous impact of different droplets facilitates the onset of splashing and instability phenomena of water surface when droplets impact on them- this can facilitate daughter droplets formation. ${ }^{49}$

As shown in the following sections, the water-soluble amphiphilic non-ionic surfactant Tween 80 is here demonstrated to facilitate the immersion and dispersion of oil in water droplets by decreasing the water/air interfacial tension. Tween 80 is also known for its excellent biocompatibility with biomolecules and proteins and its good solubility in aqueous phase, having a high HLB (i.e. hydrophile-lipophile balance) value equal to 15 . The surfactant is used in a $0 \%$ $\mathrm{v} / \mathrm{v}-3 \% \mathrm{v} / \mathrm{v}$ concentration range. Our solution container has a $300 \mu \mathrm{L}$ volume with an interfacial area of about $144 \mathrm{~mm}^{2}$ (see Figure S1). Such low surface/volume ratio is a condition typically employed in the containers used for CMC measurements where the amount of surfactant molecules at the water/air interface is negligible with respect to the number of molecules in solution, the Tween $80 \mathrm{CMC}$ resulting in values of $15-20 \mathrm{mg} / \mathrm{L} .{ }^{50-52}$ By considering that the surfactant molecular area in a surface layer is in the range of $0.4-1.3 \mathrm{~nm}^{2},{ }^{53,54}$ a total number of molecules of the order of $1 \cdot 10^{14}-3 \cdot 10^{14}$ is expected to cover the whole interfacial area of our container. Accordingly, only for surfactant concentrations $\geq 0.003 \% \mathrm{v} / \mathrm{v}$, the number of surfactant molecules in the container $\left(>10^{15}\right)$ would be enough to fully cover the air/water interface by maintaining in solution a significant number of molecules stabilizing the oil droplets. This threshold concentration corresponding to about $33 \mathrm{mg} / \mathrm{L}$ well agrees with the $\mathrm{CMC}$ values reported in previous reports. ${ }^{50-52}$ 
A

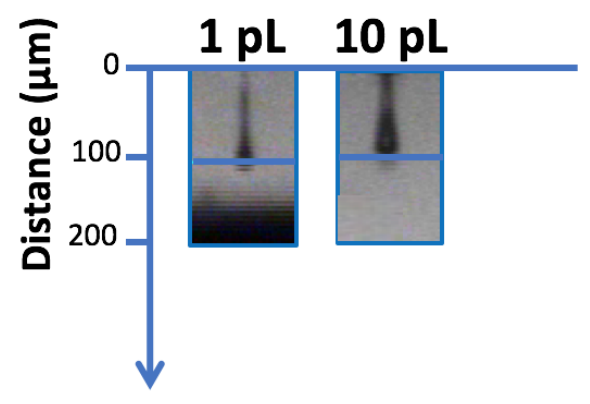

B

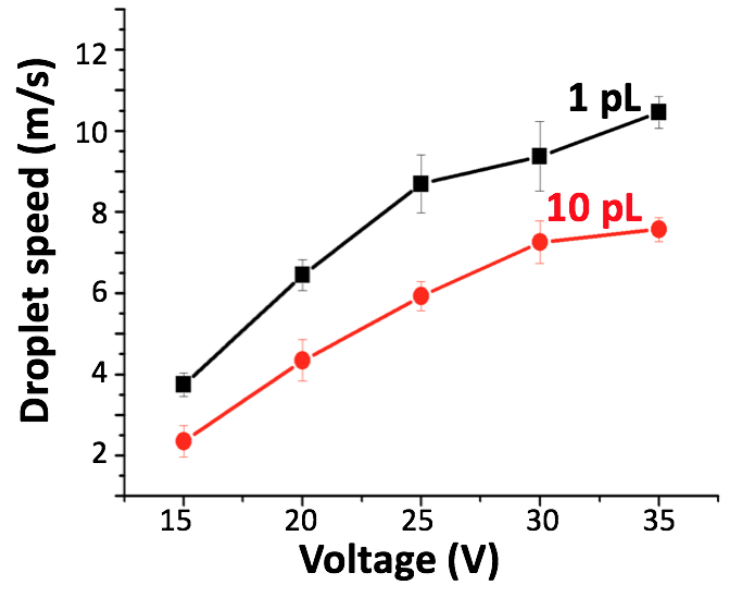

Figure 2. Inkjet printing FC40 picoliter scale oil droplets into water leads to the production of femtoliter oil-in-water droplets. A) Representative stroboscopic image of a $10 \mathrm{pL}$ and $1 \mathrm{pL}$ (nominal volume) FC40 oil droplet pinching-off at the nozzle. The formed liquid thread does not lead to satellites. B) Droplet speed as a function of jetting voltage for $10 \mathrm{pL}$ (red dots) $1 \mathrm{pL}$ (black squares) FC40 droplets.

\section{Droplet fragmentation at water-air interface lead to formation of femtoliter-scale oil droplets in aqueous phase.}

As summarized in Table 1, different experimental settings were considered, characterized by different nozzle size ( $1 \mathrm{pL}$ and $10 \mathrm{pL})$ and Tween 80 concentration $(0 \% \mathrm{v} / \mathrm{v}-3 \% \mathrm{v} / \mathrm{v})$. Note that the two different nozzle sizes are those designed for our inkjet printing device, due to the sizes of the microchannels realized on the MEMS built-up in the inkjet cartridge, which are respectively of $10.5 \mu \mathrm{m}$ and $21 \mu \mathrm{m}$ diameter.

The impedance detection chip allows for a bright field optical imaging and impedance characterization of the sample flowing through the channels. Given the significant difference in the electrical resistivity between the oil droplet (reported to be about $4.0 \times 10^{15} \mathrm{ohm} \cdot \mathrm{cm}$ by the producer) and the aqueous phase (experimentally evaluated to be about $1 \times 10^{2} \mathrm{ohm} \cdot \mathrm{cm}$ ), it is possible to successfully monitor the number and size of water-dispersed oil droplets by monitoring the impedance traces.

No droplets were optically observed neither electrically detected in case 1 pL FC40 oil droplets were printed on the aqueous phase containing 3\% v/v Tween 80 (samples S1 and S2). On the other hand, it was possible to perform optical and electrical characterizations of the FC40 droplets printed as $10 \mathrm{pL}$ droplets and dispersed in the same aqueous phase (3\% v/v Tween 80 , samples S3 and S4). Figure S7 reports a representative optical investigation of the relevant samples. It is evident that the size of a single droplet (Figure S7A) is significantly smaller in comparison to the one observed from the nozzle exit during $10 \mathrm{pL}$ droplet formation at the nozzle exit, which is not smaller than the lateral size of the nozzle $(21.5 \mu \mathrm{m}$ in the case of $10 \mathrm{pL}$ droplet). The droplets dispersed in the aqueous phase were imaged in continuous (see Supplementary video VS3). Interestingly, the size is also significantly smaller than a standard 6 $\mu \mathrm{m}$ PS bead (Figure S7B). 
Table 1. Mean electrical diameter and CV of the droplets, along with the relevant experimental conditions (ND: droplets were not detected in the impedance datastream). The presence of Tween 80 (in the range comprised between $0.003 \%$ and $3 \% \mathrm{v} / \mathrm{v}$ ) and the use of the large nozzle $(10 \mathrm{pL})$ were necessary in order to produce detectable droplets.

\begin{tabular}{lcccc} 
Sample & Nozzle size & Tween 80 & Mean diameter & CV \\
\hline \hline S1 & $1 \mathrm{pL}$ & $3 \% \mathrm{v} / \mathrm{v}$ & $\mathrm{ND}$ & $\mathrm{ND}$ \\
\hline S2 & $1 \mathrm{pL}$ & $3 \% \mathrm{v} / \mathrm{v}$ & $\mathrm{ND}$ & $\mathrm{ND}$ \\
\hline S3 & $10 \mathrm{pL}$ & $3 \% \mathrm{v} / \mathrm{v}$ & $3.2 \mu \mathrm{m}$ & $15 \%$ \\
\hline S4 & $10 \mathrm{pL}$ & $3 \% \mathrm{v} / \mathrm{v}$ & $2.9 \mu \mathrm{m}$ & $19 \%$ \\
\hline S5 & $10 \mathrm{pL}$ & $0.3 \% \mathrm{v} / \mathrm{v}$ & $3.4 \mu \mathrm{m}$ & $15 \%$ \\
\hline S6 & $10 \mathrm{pL}$ & $0.03 \% \mathrm{v} / \mathrm{v}$ & $3.3 \mu \mathrm{m}$ & $18 \%$ \\
\hline S7 & $10 \mathrm{pL}$ & $0.003 \% \mathrm{v} / \mathrm{v}$ & $2.7 \mu \mathrm{m}$ & $13 \%$ \\
\hline S8 & $10 \mathrm{pL}$ & $0.003 \% \mathrm{v} / \mathrm{v}$ & $2.8 \mu \mathrm{m}$ & $18 \%$ \\
\hline S9 & $10 \mathrm{pL}$ & $0.0003 \% \mathrm{v} / \mathrm{v}$ & $\mathrm{ND}$ & $\mathrm{ND}$ \\
\hline S10 & $10 \mathrm{pL}$ & $0.00003 \% \mathrm{v} / \mathrm{v}$ & $\mathrm{ND}$ & $\mathrm{ND}$ \\
\hline $\mathbf{S 1 1}$ & $10 \mathrm{pL}$ & - & $\mathrm{ND}$ & $\mathrm{ND}$ \\
\hline S12 & $10 \mathrm{pL}$ & - & $\mathrm{ND}$ & $\mathrm{ND}$ \\
\hline
\end{tabular}

Figure 3A reports a portion of a representative impedance data stream relevant to FC40 oil droplets (sample S3) spiked with PS beads ( $6 \mu \mathrm{m}$ size). The beads are used as internal reference to calibrate the gain factor $G$ appearing in the definition of the electrical diameter. The number of droplets/beads detected per second is also shown (Figure 3B). It is evident that FC40 oil droplets produce a much smaller electrical signal (Figure 3C) in comparison to PS bead (Figure 3D); this is accordance with the qualitative observation of the optically detected smaller size of the oil droplets in comparison with PS beads. The mean electric diameter of the droplets and its CV turned out to be $3.2 \mu \mathrm{m}$ and 15\%, respectively. As reported in Table 1, similar values were obtained also for sample $\mathrm{S} 4$, having the same Tween 80 concentration $(3 \% \mathrm{v} / \mathrm{v})$, and for the samples at lower Tween 80 concentration (S5 and S6 at $0.3 \% \mathrm{v} / \mathrm{v}$ and $0.03 \% \mathrm{v} / \mathrm{v}$, respectively, $\mathrm{S} 7$ and S8 at $0.003 \% \mathrm{v} / \mathrm{v}$ ). By contrast, it was not possible to optically observe or electrically detect FC40 oil droplets dispersed in aqueous phase if $10 \mathrm{pL}$-scale droplets were printed in aqueous phase at Tween 80 concentrations lower than $0.003 \% \mathrm{v} / \mathrm{v}$ (samples S9 and S10 at $0.0003 \% \mathrm{v} / \mathrm{v}$ and $0.00003 \% \mathrm{v} / \mathrm{v}$, respectively) or without Tween 80 (samples S11 and S12). 
A

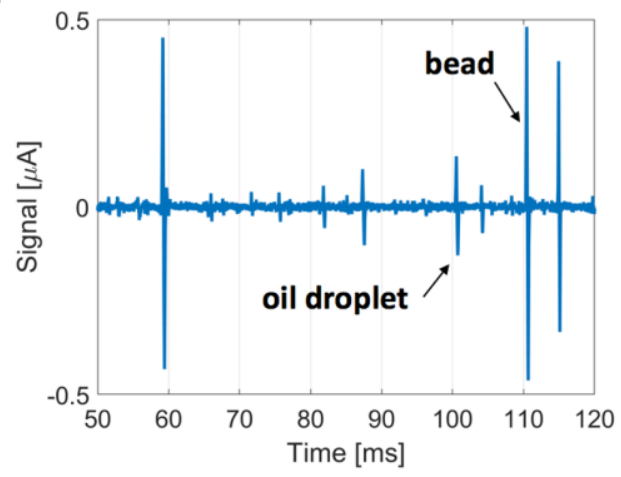

C

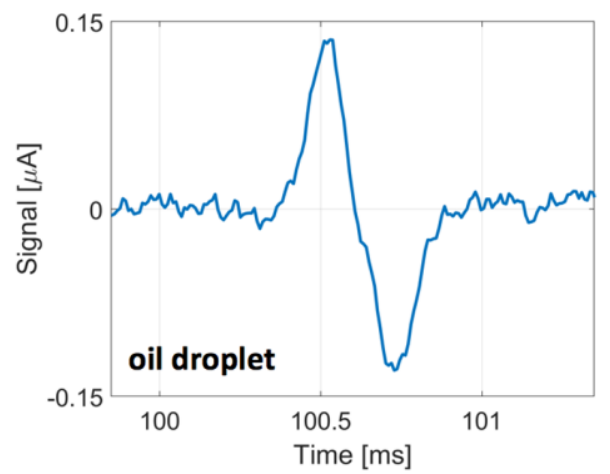

B

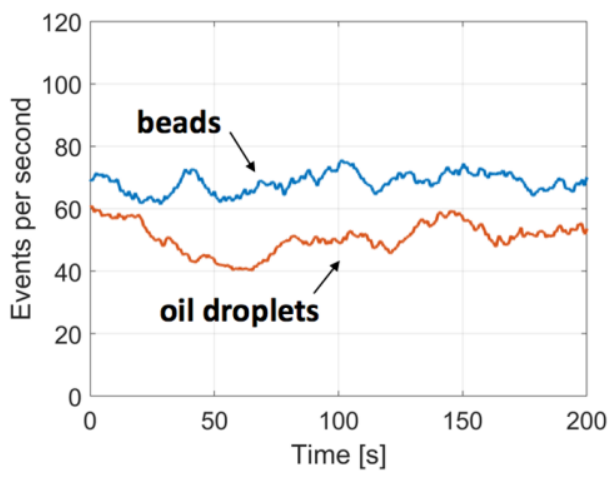

D

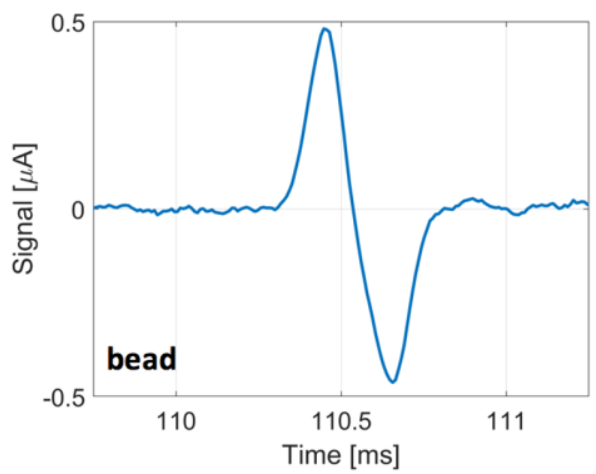

Figure 3. Single femtoliter oil droplets detected in a microfluidic chip by electrical impedance variations. A) Portion of a typical impedance data stream (i.e., real part of the differential current signal, sample S3). Two representative events relevant to an oil droplet and a $6 \mu \mathrm{m}$ bead are indicated with arrows and their zoom is plotted in panels $\mathbf{C}$ ) and D), respectively. In panel B), the number of detected droplets [resp. beads] against time is reported in red [resp. blue].

Taken together, these data are a clear indication of the fact that the fragmentation process needs the surfactant molecules coverage at the air/water interface. In particular, a crucial aspect for this dynamic is the diffusion of the surfactant to the air/water interface. This process can be described by the Ward and Tordai equation which describes the time dependence of surfactant concentration at the water/air interface. ${ }^{55} \mathrm{By}$ applying asymptotic solutions (in particular the short time approximation for $\mathrm{t} \rightarrow 0$ ), it is possible to correlate the surfactant concentration in solution with the surfactant concentration at the water/air interface as a function of time. Typically, in case of surfactant concentrations higher than CMC, the system shows a significant reduction of the surface tension with the surfactant absorption reaching a plateau value typically within $10^{3}-10^{4}$ seconds, whereas a significantly lower decrease of the surface tension is observed for surfactant concentrations lower than CMC. ${ }^{56}$ This behaviour well agrees with a picture in which the air/water surface tension declines only slightly when the surfactant coverage of the air/water interface is not higher than 60\% (Tween 80 concentrations lower than CMC), whereas it rapidly diminishes when the surfactant occupies more than $80 \%$ of the interfacial area, which occurs at values around or higher than the CMC. ${ }^{57}$ 
Figure 4 provides a schematic of the complex processes occurring in the investigated systems. In the case of pL-scale oil droplet impact onto an aqueous phase in the absence of surfactant (Figure 4A), the oil droplet is subjected to a high spread at the water/air interface (high water-air interface energy $-\sigma_{\text {water-air }}$ ) which causes its thinning at the water/air interface and subsequent evaporation. This occurs also considering that the droplet kinetic energy (of the order of $10^{-11}$ $\mathrm{Kg} \cdot \mathrm{m}^{2} \cdot \mathrm{s}^{-2}$ ) is not sufficient to overcome the water/air interfacial energy. A similar scenario occurs in case of low surfactant concentrations (lower than $0.003 \% \mathrm{v} / \mathrm{v}$ ) for which $\sigma_{\text {water-air }}$ remains high (Figure 4B). In this case there is again a high oil droplet spreading on the water surface and a lack of immersion. At lower-than-CMC tween 80 concentrations (low surfactant occupancy of the air/water interface and high interfacial tension), the oil droplets will not be able to efficiently overcome the water/air interfacial energy, and in turn the immersion process will not efficiently occur. On the other hand, in the case of a properly surfactant-laden aqueous phase,

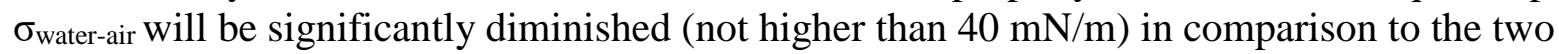
previous cases, and the oil droplet spread will be significantly compromised. Moreover, the droplet kinetic energy will be enough to overcome the water/air interfacial energy, resulting in its immersion and fragmentation (Figure 4C). 
A

pL-scale oil droplets

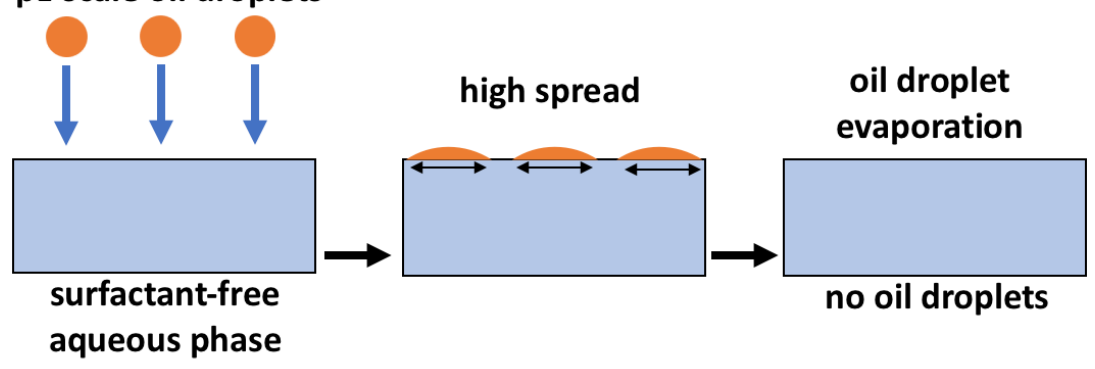

B

pL-scale oil droplets

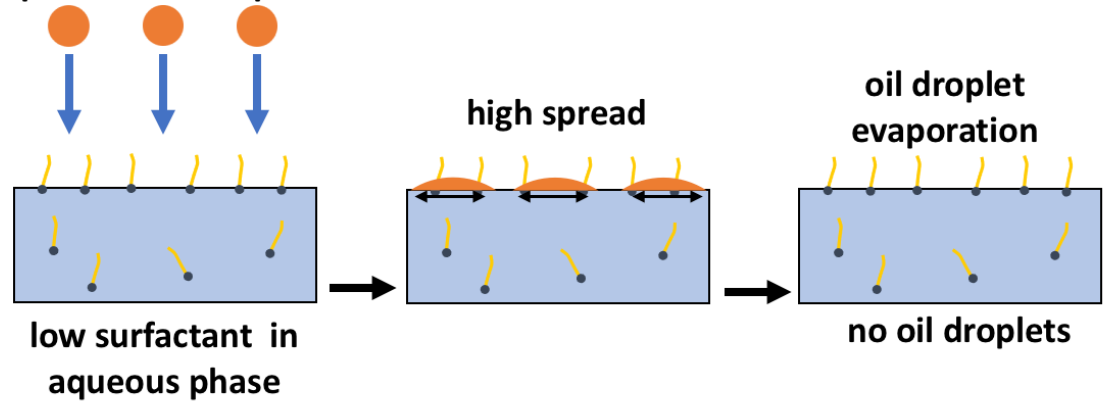

C

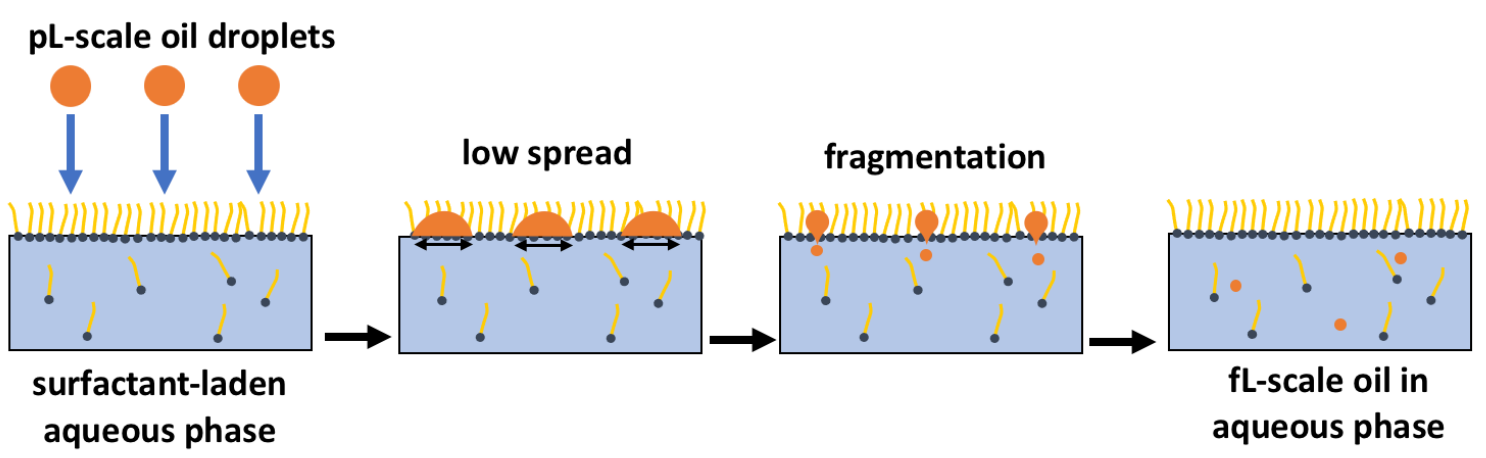

Figure 4. Spreading and immersion of pL-scale oil droplets impacting at Weber numbers $\left(\sim 10^{1}\right)$ at the surfactant-free or surfactant-laden water interfaces. (A). In the case of a surfactant-free aqueous phase, the high $\sigma_{\text {water-air }}$ causes the oil droplet to high spread and lack of immersion due to the high interfacial energy. Consequently, the spread oil droplets evaporate at the water/air interface and no daughter droplets are immersed in the aqueous phase. (B). At very low surfactant concentrations, the $\sigma_{\text {water-air }}$ will not be significantly reduced and a similar scenario will occur - i.e. pL-scale oil droplets spread with subsequent evaporation. (C). At high concentrations of surfactants, the $\sigma_{\text {water-air }}$ is significantly decreased and $\mathrm{pL}$-scale oil droplets spread is also reduced. The decreased $\sigma_{\text {water-air }}$ permits the immersion and fragmentation of pLscale oil droplets, resulting in fL-scale oil daughter droplets immersed in the aqueous phase. Evaporation occurs for the remaining part of the pL-scale oil droplets not subjected to fragmentation. 


\section{Impedance characterization of femtoliter oil-in-water droplets}

For many applications in biotechnology, it is important to add minute amounts of surfactants in order to preserve the integrity of biological structures and cells. ${ }^{58}$ In this work, the lowest surfactant concentration at which it was possible to obtain fragmented oil droplets is $0.003 \% \mathrm{v} / \mathrm{v}$ (which is of the order of its $\mathrm{CMC}^{50-52}$ ). Figure 5 reports on a representative impedance characterization of FC40 oil droplets dispersed in the aqueous phase at $0.003 \% \mathrm{v} / \mathrm{v}$ Tween 80 (sample S8). In particular, the histogram of the electrical diameter D is reported in panel A, whereas the histogram of the electrical velocity $\mathrm{V}$ in reported in panel $\mathrm{B}$, both for the droplets and the reference beads. As it can be noticed from Figure 5A, the histogram of the (monodisperse) bead population exhibits two different peaks. This is due to the positional dependence issue ${ }^{59-60}$ of impedance cytometry, combined with inertial focusing mechanisms. ${ }^{61,62}$ In fact, due to electric field non-uniformity, same size particles exhibit different electrical diameters according to their trajectory along the channel (positional dependence). In particular, with the present chip design and wiring scheme, the electric field is non-uniform across the channel height and the particles traveling at mid-height exhibit lower electrical diameter than particles traveling close to electrodes. Moreover, due to the top-bottom asymmetry caused by the cross-currents, ${ }^{63}$ the electrical diameter is higher for particles traveling near the bottom (current-collecting) electrodes than for particles traveling near the top (stimulating) electrodes. On the other hand, at particle Reynolds $\mathrm{Re}_{\mathrm{p}}$ numbers greater than one, flowing particles tend to migrate towards specific equilibrium positions in the channel cross-section due to the inertial focusing, and therefore travel along preferential trajectories. ${ }^{61,62}$ In the case of conventional straight channels, the inertial focusing is the net result of the balance of two inertial forces, i.e. the lift forces arising from the curvature of the velocity profile $\left(\mathrm{F}_{\mathrm{s}}\right.$ - i.e. the shear-induced lift $)$ and the interaction between the particle and the microchannel wall $\left(\mathrm{F}_{\mathrm{w}}\right.$ - i.e. the wall-induced lift). ${ }^{64}$ Both the two forces exhibit strong dependence upon the particle diameter a, respectively $\mathrm{F}_{\mathrm{s}} \propto \mathrm{a}^{2}$ and $\mathrm{F}_{\mathrm{w}} \propto \mathrm{a}^{3 / \delta}$, where $\delta$ is the distance to the wall. ${ }^{65,66} \mathrm{In}$ a square channel, there are four stable positions in correspondence of the middle of each wall, whereas in a rectangular channel the positions close to the short walls become unstable and only two equilibrium positions are left. $^{64}$

The two peaks in the histogram of the beads in Figure 5A correspond to two preferential particle trajectory heights. On the other hand, inertial focusing is not evident for the oil droplets, as expected due to their lower size (and hence lower $\mathrm{Re}_{\mathrm{p}}$ ). These considerations are further supported by the histograms of the electric velocity, which shows a wider range for the droplets (that visit the whole cross-section) than for the beads (that are focused). The mean electrical diameter of the droplets and its CV are reported in Table 1. As a perspective, the source of variability due to the particle trajectory could be mitigated by means of recently developed compensation strategies. ${ }^{63}$ 
A

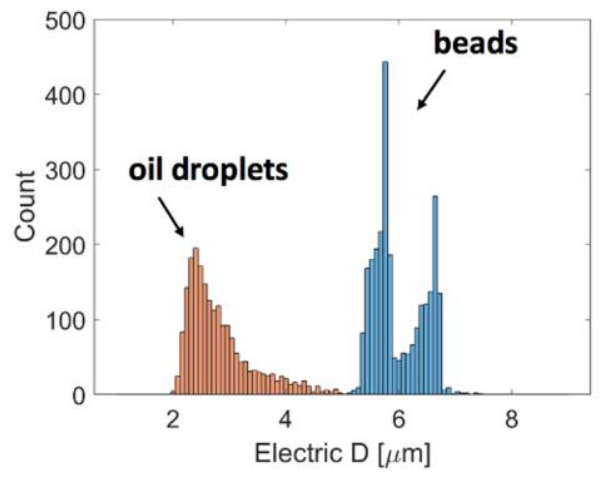

B

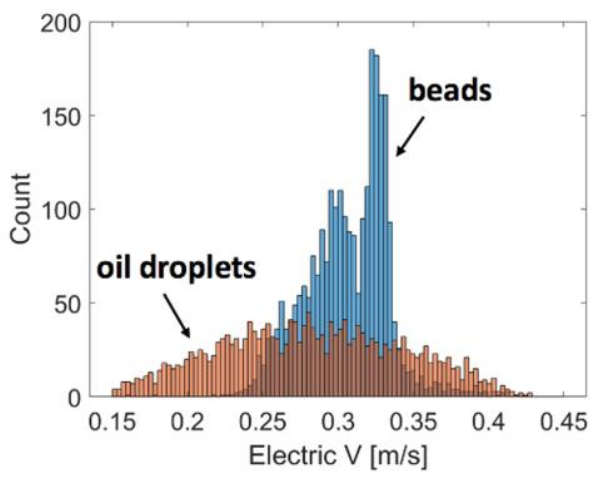

Figure 5. Representative electrical characterization of FC40 femtodroplets dispersed in aqueous phase at $0.003 \%$ v/v Tween 80 (sample S8). A) Histograms of the electrical diameter of the oil droplets (in red) and of the $6 \mu \mathrm{m}$ beads (in blue). B) Histograms of the electrical velocity of the oil droplets (in red) and of the $6 \mu \mathrm{m}$ beads (in blue). As expected, beads exhibit inertial focusing at two preferential heights. This is indicated by the presence of two peaks in their histogram of electrical diameter and by the narrow velocity range. On the other hand, oil droplets do not experience inertial focusing, due to their smaller size.

\section{Discussion}

The formation of fL-oil droplets by fragmentation at the air/water interface is based on a farfrom-equilibrium conditions by a specific sets of dynamic parameters. ${ }^{67}$ More specifically, the successful immersion of inkjetted oil droplets into water takes into account a quite complex dynamics at water/air interface involving oil droplet impact deformation on surfactant-rich water/oil interface, spreading, droplet breakups and immersion in the aqueous phase.$^{68} \mathrm{Immersed}$ oil droplets are then stabilized by the presence of the surfactant.

Droplet impact: oil-in-water vs. water-in-oil. The interaction of a drop into an immiscible system is a topic of great interest not only from the fundamental understanding of the physico-chemical phenomena but also for the numerous technological applications such as surface coating, spray cooling, thin polymer films wrap up drop encapsulation. ${ }^{31}$ In particular, studies mainly consider two possible impact scenarios: oil drop onto an aqueous phase ${ }^{31}$ or water drop onto an oil phase. ${ }^{28}$ The final outcome of these two set-ups is very different.

The impact of an oil droplet on water (considering water thickness much higher than droplet diameter) results in a deformation of the water surface giving rise to a crown and capillary waves, which propagate outward on the liquid film. ${ }^{49}$ After crown collapsing, oil droplet spreads on water surface on the time scale of milliseconds. ${ }^{69}$ This can be quantified by considering the spreading factor:

$\mathrm{S}=\sigma_{\text {water-air }}-\left(\sigma_{\text {oil-air }}+\sigma_{\text {water-oil }}\right)$

In which $\sigma_{\text {water-air }}$ is the water-air interface energy, $\sigma_{\text {oil-air }}$ is the oil-air interface energy (around 11$12 \mathrm{mN} / \mathrm{m}$ ), $\sigma_{\text {water-oil }}$ is the water-oil interface energy (typically around $20 \mathrm{mN} / \mathrm{m}$ ). More specifically, the spreading factor of a FC40 oil droplet on water surface is always positive - i.e. upon impact of the oil droplet with the water film, the difference in surface tension is a driving force to spread the oil droplet on the water film. In particular, the higher is the values of $\sigma_{\text {water-air, }}$, the higher will be the oil droplet spreading and hence, the thinner will be oil film on the surface. Accordingly, the presence of surfactant molecules in the aqueous phase is known to reduce the 
thinning of oil droplet on the water surface, because upon impact, the outward flow of air and water lead to an extension of the surface area covered by the surfactant, reducing the local surface concentration of Tween 80 which, in turn, leads to higher surface tension and Marangoni stress. $^{31}$

The opposite scenario, namely water-in-oil impact, is associated with a negative spreading factor which indicates that water droplet does not spread on the oil surface, given its higher surface tension. In particular, the interaction of a water drop onto an oil phase leads to the formation of a crown followed by a central jet. More specifically, the crown is a consequence of the drop impact on the oil. While the crown propagates radially outwards, capillarity forces emerge, causing the crown collapse onto the film and generating capillary waves which propagate radially on the film surface away from the impact point. As a result, capillary causes droplet retraction which forces the motion of the droplet towards the impact point leading to the formation of a liquid jet. After the dynamics of impact, since the water droplet has a higher density than the oil phase, an engulfment of the water droplet into the oil droplet will occur as shown in previous reports. ${ }^{70,28}$

Accordingly, this implies that, in contrast to the rich dynamics of oil-in-water system, a pL-scale water droplet impacting on oil surface can ultimately adopt an equilibrium, lens-like shape without any fragmentation phenomena. ${ }^{28}$ We experimentally verified this by printing single 10 pL scale water droplets containing a fluorolabeled dye into mineral oil at conditions similar to those of oil droplets (Figure S8).

Droplet fragmentation and immersion. The spread oil droplet at the water-air interface is known to create significant surface tension gradients, in particular decrements of the water-air surface tension which are able to trigger surface oil droplets instability due to the spontaneous formation of capillary waves ${ }^{71}$ which can lead to oil breakup in resulting daughter droplets having sizes which can be as low as 10-20\% of the initial droplet size according to experimental investigations on macroscale droplets, being the number and the size of the resulting droplets dependent from the Weber number of the droplet. ${ }^{33}$ As reported in the results, $10 \mathrm{pL}$ inkjetted droplets gave rise to immersed oil droplets as a result of droplet fragmentation. On the other hand, it was not possible to detect immersed droplets derived from inkjetted $1 \mathrm{pL}$ droplets. This result is unexpected because the values of the Weber number for both $1 \mathrm{pL}$ and $10 \mathrm{pL}$ droplets are comparable (around 50 and 70, respectively) and are known to lead, according to Lhuissier et al. ${ }^{33}$ to droplet immersion resulting in axial fragmentation regime upon impact with water surface for which the immersed drop shapes into a single ligament and fragments into few droplets (even up to single droplet) along the (vertical) axis of symmetry. ${ }^{33}$ Nevertheless, the result can be ascribed to the lower tendency of thinner spread oil film formed by $1 \mathrm{pL}$ droplet (in comparison with $10 \mathrm{pL}$ ) to give rise to capillary instabilities ${ }^{71}$ which, in turn, leads to significantly fewer immersed oil droplets in aqueous phase or, more simply to the small size of the resulting daughter droplets (around 1-2 microns if scaled according to the sizes of resulting daughter droplets derived from $10 \mathrm{pL}$ inkjetted droplets) which would be almost at the limit of resolution for the present impedance chip.

Interestingly, the observed droplet size distribution of oil immersed droplets derived from $10 \mathrm{pL}$ inkjetted droplets (Figure 5B) resembles the shape predicted by theoretical models based on macroscopic droplets ${ }^{33}$ according to which the normalized distribution of droplet sizes goes with $(8 / 9) \cdot(d /\langle d\rangle)^{-4}-$ i.e. the droplet size rapidly decreases from the average down to larger values. The distribution is independent from the Weber number and can be considered as a universal law valid also under different dynamic regimes. The CV of droplets diameters (around 13\%-19\%) is 
comparable to those obtainable from conventional emulsification approaches.${ }^{14}$ Remarkably, the number of droplets produced from the fragmentation process is not a direct result of the total size of the inkjetted droplet - however, in accordance to models of droplet axial fragmentation at these Weber number (around $10^{1}$ ), this number is only partially correlated to the number of droplets which impact on the aqueous phase, because not all fragmented droplets remain in the receiving aqueous phase but are ejected in the air, with consequent droplet evaporation.

Surfactant effect on the droplet immersion and stability. As previously reported by Che et al., the dynamics of droplet impact on a miscible liquid is significantly altered by the presence of surfactant molecules following a quite complex dynamics ${ }^{31}$ which depends on the surfactant presence in the droplet and/or in the receiving phase. In the case of surfactant-laden droplets, Marangoni stresses on the surface of the liquid film can enhance the capillary wave propagation on the surface of the receiving liquid. On the other hand, in the case of the droplet impact onto the surface of a surfactant-laden liquid, the spreading of the droplet is partially inhibited because of the reduction of $\sigma_{\text {water-air. }}{ }^{31}$ These observations permit to better elucidate the fundamental mechanisms played by the surfactant molecules during the impact and the subsequent immersion of fragmented oil droplets into the aqueous phase. Remarkably, droplet downsizing from macroscale to microscale plays an important role in this regard.

Whereas macroscale droplets which have a Bond number approaching 1 (defined as $B_{0}=\frac{\rho g l^{2}}{\sigma}$, where $\rho\left[\mathrm{Kg} / \mathrm{m}^{3}\right]$ is the density, $g\left[\mathrm{~m} / \mathrm{s}^{2}\right]$ is the gravity acceleration, $l[\mathrm{~m}]$ is the characteristic length and $\sigma[\mathrm{N} / \mathrm{m}]$, is the surface tension), microscale droplets are characterized by a significantly lower Bond number $\left(10^{-4}-10^{-5}\right)$, and thus the droplet immersion is not dependent by gravitational contribution but quite exclusively by surface tension forces. It is important to note that in order for the spread oil droplet to be immersed in the aqueous phase, its kinetic energy has to overcome the water/air interfacial energy, ${ }^{11}$ otherwise it fails to separate from the water-air interface, with consequent complete spreading on the interface and evaporation which can be estimated to occur on the hundreds of milliseconds time scale.

In the current reports dealing with macroscale droplets impact on fluids, surfactant effect is not critical for the oil droplet immersion in the aqueous phase. ${ }^{31}$ It is known that the presence of surfactants is able to mitigate the speed of the capillary waves because of the reduction of $\sigma_{\text {water- }}$ air which would in principle decrease the onset of oil droplets fragmentation.

On the other hand, in the case of micron-sized oil droplets emulsification produced by inkjet printing, the presence of the surfactant is essential. In fact, it was not possible to detect any oil droplets when immersing inkjetted droplets in the surfactant-free aqueous phase. This result can be explained as a consequence of the coalescence of FC40 oil droplets once immersed in the aqueous phase when in the absence of surfactant. Indeed, whereas the dynamics of droplet fragmentation can benefit by the lack of Tween 80 at the water/air interface due to the triggering of higher speed capillary waves, on the other hand the stabilization of immersed micron-sized droplets strictly depends upon the presence of surfactant agent in the aqueous phase, as a remarkable difference with respect to macroscale daughter droplets. In addition, it is not possible to exclude that in the absence of Tween 80, FC40 oil droplet is subjected to a more significant thinning on the water surface with respect to the surfactant-rich surface due to the higher spreading factor. The higher oil thinning can finally reduce the thickness of the oil film and thus, similarly to $1 \mathrm{pL}$ droplets, the possibility to generate capillary waves and then daughter droplets in the aqueous phase.

\section{Conclusions}


This study reports an unprecedented droplet fragmentation based approach for the production of surfactant stabilized fL-scale oil droplets dispersed in the aqueous phase from the direct inkjet printing of pL-scale droplets in the aqueous phase. The success in the direct immersion of micron-sized oil droplet in an aqueous phase is strictly dependent on two factors: the density of the oil phase has to be sufficiently large in comparison to the density of the aqueous phase; the droplet kinetic energy has to be high enough to overcome the opposing water/air interfacial barrier. In the specific case of oil-in-water emulsions, electrical impedance allows for the detection of oil droplets at the single level, permitting to count the number of oil droplets per volume and characterize their diameter with size detection limit up to few microns. The oil droplets can be formed at low concentrations of non-ionic mild Tween 80 surfactant (as low as $0.003 \% \mathrm{v} / \mathrm{v}$ ) which opens interesting perspectives for applications where the surfactant concentrations have to be kept as low as possible. The oil droplet sizes (typically around 3 microns range) are readily measured by electrical impedance at rates as high as some tens of events/second. In principle, this fragmentation method can be extended to other liquid/liquid systems, by meeting the above defined criteria - i.e. the density and surface tension gradient between the two different phases. The study presented herein is a step forward to the highthroughput, low cost, low energy consumption fabrication of fL-scale oil-in-water emulsions. These results can find immediate applications for the scalable encapsulation of fluids by surfactant thin films to produce at high speed and in an automatable approach, isolated oil subcellular scale microcompartments dispersed in an immiscible continuous phase. In addition, a more in-depth control of the droplet dynamics during the fragmentation phenomena at the water/air interface might permit to achieve droplets of different sizes, like for instance the same size of the inkjetted droplet at lower We, or higher number of fragmented droplets at significantly higher We $\left(10^{2}\right)$ resulting from azimuthal droplets, allowing a truly scalable and tailored production of droplets in immiscible phases.

\section{ASSOCIATED CONTENT}

\section{Supporting Information.}

The following files are available free of charge on the ACS Publications website at DOI: xxx

Complementary figures (PDF)

Video recording of flowing droplets (avi)

\section{AUTHOR INFORMATION}

\section{Corresponding Authors}

* Email: (Giuseppe Arrabito) giuseppedomenico.arrabito@unipa.it, (Federica Caselli) caselli@ing.uniroma2.it

\section{Author Contributions}

G.A. and F.Cas. conceived the original idea and developed the experimental design. G.A., F.Cav. and V.F. prepared the oil droplets. V.E. and A.d.N. carried out electrical characterizations on droplets. G.A. and F.Cas. analysed the data. F.Cas. and B.P. planned the experiments and coordinated the entire work. The manuscript was written through contributions of all authors. All authors have given approval to the final version of the manuscript. 


\section{ACKNOWLEDGMENT}

The research leading to this work was supported by the SPY Project (E81I18000540005), funded under the "Mission Sustainability Programme" of the University of Rome Tor Vergata. The laboratories of ATeN center (www.atencenter.com) are also acknowledged for hospitality and support. Prof. G. Marletta (Department of Chemical Sciences, University of Catania) and Prof. P. Bisegna (Department of Civil Engineering and Computer Science, University of Rome Tor Vergata) are acknowledged for thoughtful discussions and useful comments provided during drafting the manuscript. Prof.ssa V. Vetri (Department of Physics and Chemistry, University of Palermo) is acknowledged for confocal microscopy fluorescence images of aqueous droplets printed in mineral oil drops.

\section{REFERENCES}

(1) Marquez, R.; Forgiarini, A. M.; Langevin, D.; Salager, J. L. Instability of Emulsions Made with Surfactant-Oil-Water Systems at Optimum Formulation with Ultralow Interfacial Tension. Langmuir 2018, 34, 9252-9263.

(2) McClements, D. J. Nanoemulsions versus Microemulsions: Terminology, Differences, and Similarities. Soft Matter 2012, 8, 1719-1729.

(3) Berton-Carabin, C. C.; Schroën, K. Pickering Emulsions for Food Applications:

Background, Trends, and Challenges. Annu. Rev. Food Sci. Technol. 2015, 6, 263-297.

(4) Theberge, A. B.; Courtois, F.; Schaerli, Y.; Fischlechner, M.; Abell, C.; Hollfelder, F.; Huck, W. T. S. Microdroplets in Microfluidics: An Evolving Platform for Discoveries in Chemistry and Biology. Angew. Chemie - Int. Ed. 2010, 49, 5846-5868.

(5) Stolovicki, E.; Ziblat, R.; Weitz, D. A. Throughput Enhancement of Parallel Step Emulsifier Devices by Shear-Free and Efficient Nozzle Clearance. Lab Chip 2017, 18, $132-138$.

(6) Jaiswal, M.; Dudhe, R.; Sharma, P. K. Nanoemulsion: An Advanced Mode of Drug Delivery System. 3 Biotech 2015, 5, 123-127.

(7) Sivakumar, B. S.; Bansal, V.; Cortez, C.; Chong, S.; Zelikin, A. N.; Caruso, F. PolymerEncapsulated Emulsions as Anticancer Drug Carriers. Adv. Mater. 2009, 3010, 18201824.

(8) Toyota, T.; Maru, N.; Hanczyc, M. M.; Ikegami, T.; Sugawara, T. Self-Propelled Oil Droplets Consuming "Fuel” Surfactant. J. Am. Chem. Soc. 2009, 131, 5012-5013.

(9) Hirono, A.; Toyota, T.; Asakura, K.; Banno, T. Locomotion Mode of Micrometer-Sized Oil Droplets in Solutions of Cationic Surfactants Having Ester or Ether Linkages. Langmuir 2018, 34, 7821-7826. 
(10) Caschera, F.; Rasmussen, S.; Hanczyc, M. M. An Oil Droplet Division-Fusion Cycle. Chempluschem 2013, 78, 52-54.

(11) Kumar, D.; Paulsen, J. D.; Russell, T. P.; Menon, N. Wrapping with a Splash: High-Speed Encapsulation with Ultrathin Sheets. Science 2018, 359, 775-778.

(12) Sahin, S.; Bliznyuk, O.; Rovalino Cordova, A.; Schroën, K. Microfluidic EDGE Emulsification: The Importance of Interface Interactions on Droplet Formation and Pressure Stability. Sci. Rep. 2016, 6, 26407.

(13) Nakashima, T.; Shimizu, M. Preparation of Monodispersed O/W Emulsion by Porous Glass Membrane. Kagaku Kogaku Ronbunshu 1993, 19, 984-990.

(14) Kawakatsu, T.; Kikuchi, Y.; Nakajima, M. Regular-Sized Cell Creation in Microchannel Emulsification by Visual Microprocessing Method. J. Am. Oil Chem. Soc. 1997, 74, 317321.

(15) Liu, W.; Yang, X.-L.; Winston Ho, W. S. Preparation of Uniform-Sized Multiple Emulsions and Micro/Nano Particulates for Drug Delivery by Membrane Emulsification. J. Pharm. Sci. 2010, 100, 75-93.

(16) Chung, B. G.; Lee, K.-H.; Khademhosseini, A.; Lee, S.-H. Microfluidic Fabrication of Microengineered Hydrogels and Their Application in Tissue Engineering. Lab Chip 2012, $12,45-59$.

(17) Hansen, M. M. K.; Meijer, L. H. H.; Spruijt, E.; Maas, R. J. M.; Rosquelles, M. V.; Groen, J.; Heus, H. A.; Huck, W. T. S. Macromolecular Crowding Creates Heterogeneous

Environments of Gene Expression in Picolitre Droplets. Nat Nanotechnol. 2016, 11, 191197.

(18) Mastrobattista, E.; Taly, V.; Chanudet, E.; Treacy, P.; Kelly, B. T.; Griffiths, A. D. HighThroughput Screening of Enzyme Libraries: In Vitro Evolution of a $\beta$-Galactosidase by Fluorescence-Activated Sorting of Double Emulsions. Chem. Biol. 2005, 12, 1291-1300.

(19) Sugiura, S.; Nakajima, M.; Kumazawa, N.; Iwamoto, S.; Seki, M. Characterization of Spontaneous Transformation-Based Droplet Formation during Microchannel Emulsification. J. Phys. Chem. B 2002, 106, 9405-9409.

(20) Kobayashi, I.; Neves, M. A.; Wada, Y.; Uemura, K.; Nakajima, M. Large Microchannel Emulsification Device for Producing Monodisperse Fine Droplets. Procedia Food Sci. 2011, 1, 109-115.

(21) Liao, S.; Tao, Y.; Du, W.; Wang, Y. Interfacial Emulsification: An Emerging Monodisperse Droplet Generation Method for Microreactors and Bioanalysis. Langmuir 2018, 34, 11655-11666.

(22) Arrabito, G.; Musumeci, C.; Aiello, V.; Libertino, S.; Compagnini, G.; Pignataro, B. On the Relationship between Jetted Inks and Printed Biopatterns: Molecular-Thin Functional 
Microarrays of Glucose Oxidase. Langmuir 2009, 25, 6312-6318.

(23) Arrabito, G.; Reisewitz, S.; Dehmelt, L.; Bastiaens, P. I.; Pignataro, B.; Schroeder, H.; Niemeyer, C. M. Biochips for Cell Biology by Combined Dip-Pen Nanolithography and DNA-Directed Protein Immobilization. Small 2013, 9, 4243-4249.

(24) Arrabito, G.; Schroeder, H.; Schröder, K.; Filips, C.; Marggraf, U.; Dopp, C.; Venkatachalapathy, M.; Dehmelt, L.; Bastiaens, P. I. H.; Neyer, A.; et al. Configurable Low-Cost Plotter Device for Fabrication of Multi-Color Sub-Cellular Scale Microarrays. Small 2014, 10, 2870-2876.

(25) Gandor, S.; Reisewitz, S.; Venkatachalapathy, M.; Arrabito, G.; Reibner, M.; Schröder, H.; Ruf, K.; Niemeyer, C. M.; Bastiaens, P. I. H.; Dehmelt, L. A Protein-Interaction Array Inside a Living Cell. Angew. Chemie Int. Ed. 2013, 52, 4790-4794.

(26) Arrabito, G.; Pignataro, B. Solution Processed Micro- and Nano-Bioarrays for Multiplexed Biosensing. Anal. Chem. 2012, 84, 5450-5462.

(27) Miccichè, C.; Arrabito, G.; Amato, F.; Buscarino, G.; Agnello, S.; Pignataro, B. Inkjet Printing Ag Nanoparticles for SERS Hot Spots. Anal. Methods 2018, 10, 3215-3223.

(28) Arrabito, G.; Cavaleri, F.; Montalbano, V.; Vetri, V.; Leone, M.; Pignataro, B. Monitoring Few Molecular Binding Events in Scalable Confined Aqueous Compartments by Raster Image Correlation Spectroscopy (CADRICS). Lab Chip 2016, 16, 4666-4676.

(29) Arrabito, G.; Galati, C.; Castellano, S.; Pignataro, B. Luminometric Sub-Nanoliter Droplet-to-Droplet Array (LUMDA) and Its Application to Drug Screening by Phase i Metabolism Enzymes. Lab Chip 2013, 13, 68-72.

(30) Zeng, H.; Yang, J.; Katagiri, D.; Rang, Y.; Xue, S.; Nakajima, H.; Uchiyama, K. Investigation of Monodisperse Droplet Generation in Liquids by Inkjet. Sensors Actuators B Chem. 2015, 220, 958-961.

(31) Che, Z.; Matar, O. K. Impact of Droplets on Liquid Films in the Presence of Surfactant. Langmuir 2017, 33, 12140-12148.

(32) Che, Z.; Matar, O. K. Impact of Droplets on Immiscible Liquid Films. Soft Matter 2018, 14, 1540-1551.

(33) Lhuissier, H.; Sun, C.; Prosperetti, A.; Lohse, D. Drop Fragmentation at Impact onto a Bath of an Immiscible Liquid. Phys. Rev. Lett. 2013, 110, 2-6.

(34) Cheung, K. C.; Di Berardino, M.; Schade-Kampmann, G.; Hebeisen, M.; Pierzchalski, A.; Bocsi, J.; Mittag, A.; Tárnok, A. Microfluidic Impedance-Based Flow Cytometry. Cytom. Part A 2010, 77, 648-666.

(35) Petchakup, C.; Li, K. H. H.; Hou, H. W. Advances in Single Cell Impedance Cytometry for Biomedical Applications. Micromachines 2017, 8, 87. 
(36) Rollo, E.; Tenaglia, E.; Genolet, R.; Bianchi, E.; Harari, A.; Coukos, G.; Guiducci, C. Label-Free Identification of Activated $\{\mathrm{T}\}$-Lymphocytes through Tridimensional Microsensors on Chip. Biosens. Bioelectron. 2017, 94, 193-199.

(37) McGrath, J. S.; Honrado, C.; Spencer, D.; Horton, B.; Bridle, H. L.; Morgan, H. Analysis of Parasitic Protozoa at the Single-Cell Level Using Microfluidic Impedance Cytometry. Sci. Rep. 2017, 7.

(38) Yakdi, N. E.; Huet, F.; Ngo, K. Detection and Sizing of Single Droplets Flowing in a Labon-a-Chip Device by Measuring Impedance Fluctuations. Sensors Actuators B Chem. 2016, 236, 794-804.

(39) Kalantarifard, A.; Saateh, A. Label-Free Sensing in Microdroplet-Based Microfluidic Systems. Chemosensors 2018, 6, 23.

(40) Klibanov, A. L.; Hossack, J. A. Lab on a Chip A Flow Focusing Microfluidic Device with an Integrated Coulter Particle Counter for Production, Counting and Size Characterization of Monodisperse Microbubbles. Lab Chip 2018, 18, 2653-2664.

(41) Sun, T.; Morgan, H. Single-Cell Microfluidic Impedance Cytometry: A Review. Microfluid. Nanofluid. 2010, 8, 423-443.

(42) Caselli, F.; Bisegna, P. A Simple and Robust Event-Detection Algorithm for Single-Cell Impedance Cytometry. IEEE Trans Biomed Eng 2016, 63, 415-422.

(43) Errico, V.; De Ninno, A.; Bertani, F.; Businaro, L.; Bisegna, P.; Caselli, F. Mitigating Positional Dependence in Coplanar Electrode Coulter-Type Microfluidic Devices. Sens. Act. B Chem. 2017, 247, 580-586.

(44) De Ninno, A.; Errico, V.; Bertani, F. R.; Businaro, L.; Bisegna, P.; Caselli, F. Coplanar Electrode Microfluidic Chip Enabling Accurate Sheathless Impedance Cytometry. Lab Chip 2017, 17, 1158-1166.

(45) Clausen, H. C.; Dimaki, M.; Bertelsen, V. C.; Skands, E. G.; Rodriguez-Trujillo, R.; Thomsen, D. J.; Svendsen, E. W. Bacteria Detection and Differentiation Using Impedance Flow Cytometry. Sensors 2018, 18, 3496.

(46) Esfandyarpour, R.; Javanmard, M.; Koochak, Z.; Harris, J. S.; Davis, R. W. Nanoelectronic Impedance Detection of Target Cells. Biotechnol. Bioeng. 2014, 111, 1161-1169.

(47) Holt, D. J.; Payne, R. J.; Chow, W. Y.; Abell, C. Fluorosurfactants for Microdroplets: Interfacial Tension Analysis. J. Colloid Interface Sci. 2010, 350, 205-211.

(48) Dong, H.; Carr, W. W.; Morris, J. F. An Experimental Study of Drop-on-Demand Drop Formation. Phys. Fluids 2006, 18, 072102.

(49) Liang, G.; Zhang, T.; Yu, H.; Chen, H.; Shen, S. Simultaneous Impact of Multiple 
Droplets on Liquid Film. J. Ind. Eng. Chem. 2018, 65, 51-61.

(50) Mandal, A. B.; Nair, B. U.; Ramaswamy, D. Determination of the Critical Micelle Concentration of Surfactants and the Partition Coefficient of an Electrochemical Probe by Using Cyclic Voltammetry. Langmuir 1988, 4, 736-739.

(51) Andreatta, G.; Bostrom, N.; Mullins, O. C. High-Q Ultrasonic Determination of the Critical Nanoaggregate Concentration of Asphaltenes and the Critical Micelle Concentration of Standard Surfactants. Langmuir 2005, 21, 2728-2736.

(52) Mahmood, M. E.; Al-koofee, D. A. F. Effect of Temperature Changes on Critical Micelle Concentration for Tween Series Surfactant. Glob. J. Sci. Front. Res. Chem. 2013, 13, 1-7.

(53) Tang, X.; Huston, K. J.; Larson, R. G. Molecular Dynamics Simulations of StructureProperty Relationships of Tween 80 Surfactants in Water and at Interfaces. J. Phys. Chem. B 2014, 118, 12907-12918.

(54) Kareemand, S. H.; Ali, B. Surface Properties of Mixed Adsorbed Surfactants Film of Tween 20 and Tween 80 on Liquid - Air Interfacial. Int. J. Sci. Res. 2017, 6, 2015-2018.

(55) Eastoe, J.; Dalton, J. S. Dynamic Surface Tension and Adsorption Mechanisms of Surfactants at the Air-Water Interface. Adv. Colloid Interface Sci. 2000, 85, 103-144.

(56) Kramek-romanowska, K.; Sosnowski, T. R. Surface Tension Reduction in Air-Water Systems Containing Nonionic Surfactant - Comparison of Mass Transfer Limited Models. Inżynieria i Apar. Chem. 2017, 56, 78-79.

(57) Menger, F. M.; Rizvi, S. A. A. Relationship between Surface Tension and Surface Coverage. Langmuir 2011, 27, 13975-13977.

(58) Chen, C.-M.; Chou, H.-C.; Lin, W.; Tseng, C. Surfactant Effects on the Viability and Function of Human Mesenchymal Stem Cells: In Vitro and in Vivo Assessment. Stem Cell Res. Ther. 2017, 8, 180.

(59) Spencer, D.; Morgan, H. Positional Dependence of Particles in Microfludic Impedance Cytometry. Lab Chip 2011, 11, 1234-1239.

(60) Spencer, D.; Caselli, F.; Bisegna, P.; Morgan, H. High Accuracy Particle Analysis Using Sheathless Microfluidic Impedance Cytometry. Lab Chip 2016, 16, 2467-2473.

(61) Di Carlo, D. Inertial Microfluidics. Lab Chip 2009, 9, 3038-3046.

(62) Reale, R.; Ninno, A. De; Businaro, L.; Bisegna, P.; Caselli, F. Electrical Measurement of Cross - Sectional Position of Particles Flowing through a Microchannel. Microfluid. Nanofluidics 2018, 22, 1-13.

(63) Caselli, F.; De Ninno, A.; Reale, R.; Businaro, L.; Bisegna, P. A Novel Wiring Scheme for Standard Chips Enabling High-Accuracy Impedance Cytometry. Sensors Actuators B 
Chem. 2018, 256, 580-589.

(64) Zhou, J.; Papautsky, I. Lab on a Chip Fundamentals of Inertial Focusing in Microchannels. Lab Chip 2013, 13, 1121-1132.

(65) Loth, E.; Dorgan, A. J. An Equation of Motion for Particles of Finite Reynolds Number and Size. Environ. Fluid Mech. 2009, 9, 187-206.

(66) Stephen Williams, P.; Lee, S.; Calvin Giddings, J. Characterization of Hydrodynamic Lift Forces by Field-Flow Fractionantion. Inertial and Near-Wall Lift Forces. Chem. Eng. Commun. 1994, 130, 143-166.

(67) Fabiano, S.; Pignataro, B. Selecting Speed-Dependent Pathways for a Programmable Nanoscale Texture by Wet Interfaces. Chem. Soc. Rev. 2012, 41, 6859-6873.

(68) Eggers, J.; Villermaux, E. Physics of Liquid Jets. Reports Prog. Phys. 2008, 71.

(69) Sanatkaran, N.; Kulichikhin, V. G.; Malkin, A. Y.; Foudazi, R. Spreading of Oil-in-Water Emulsions on Water Surface. Langmuir 2018, 34, 10974-10983.

(70) Sun, Y.; Chen, X.; Zhou, X.; Zhu, J.; Yu, Y. Droplet-in-Oil Array for Picoliter-Scale Analysis Based on Sequential Inkjet Printing. Lab Chip 2015, 15, 2429-2436.

(71) Valkovska, D. S.; Kralchevsky, P. A.; Danov, K. D.; Broze, G.; Mehreteab, A. Effect of Oil Solubility on the Oil Drop Entry at Water-Air Interface. Langmuir 2000, 16, 88928902. 